\title{
Selective Oxidation of Zirconocyclopentenes via Organoboranes
}

\author{
Jeffrey S. T. Gorman, Scott T. lacono and Brian L. Pagenkopf* \\ Department of Chemistry and Biochemistry, The University of Texas at Austin, \\ Austin, TX 78712 \\ Pagenkopf@mail.utexas.edu
}

Experimental Section

All reactions were run under an atmosphere of nitrogen or argon. Flasks were oven or flamedried and allowed to cool in a desiccator prior to use. Solvents and reagents were purified by standard methods. ${ }^{1}$ Thin-layer chromatography (TLC) was performed on EM 250 Kieselgel 60 F254 silica gel plates. The plates were visualized by staining with $\mathrm{I}_{2}$ on silica, $\mathrm{CAM},{ }^{2}$ or potassium permanganate. GC analyses were performed on a Hewlett-Packard 5890 Series II instrument equipped with an Econocap column (Altech, $15 \mathrm{~m} \mathrm{x} 0.53 \mu \mathrm{m}$ ) and FID detector.

The ${ }^{1} \mathrm{H}$ and ${ }^{13} \mathrm{C}$ NMR data was obtained on a Varian Unity Plus 300 or a Varian INOVA 400 or 500 spectrometer. For ${ }^{1} \mathrm{H}$ NMR, chemical shifts are reported in parts per million (ppm) downfield from tetramethylsilane and are, in all cases, referenced to the residual proton resonance peaks: $\delta 7.24$ for $\mathrm{CHCl}_{3}$. The ${ }^{13} \mathrm{C} \mathrm{NMR}$ chemical shifts were reported in ppm relative to the center peak of the solvent multiplet: $\delta 77.0(\mathrm{t})$ for $\mathrm{CDCl}_{3} \cdot{ }^{13} \mathrm{C}$ NMR spectra were routinely run with broadband ${ }^{1} \mathrm{H}$ decoupling. NOE differences were determined using a double pulsed field gradient spin echo sequence (mixing time $=800 \mathrm{msec}$ ) and values are reported adjacent to the enhanced hydrogen(s). Infrared spectra were obtained on a Nicolet AVATAR 360 FTIR. HRMS (CI) were made with a VG analytical ZAB2-E instrument. Where appropriate, descriptions of signals include broad (br), apparent (app) and multiplet (m).

Enynes in entries $\mathbf{3}^{3}, \mathbf{5}^{4}, \mathbf{8}^{4}$ were prepared according to previously described procedures.

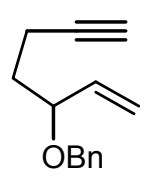

\section{3-Benzyloxyhept-1-en-6-yne.}

To a suspension of $\mathrm{NaH}(0.31 \mathrm{~g}, 94 \%, 12 \mathrm{mmol})$ in $\mathrm{DMF}(11 \mathrm{~mL})$ at $0{ }^{\circ} \mathrm{C}$ 3-hydroxyhept1 -en-6-yne ${ }^{5}(1.20 \mathrm{~g}, 11 \mathrm{mmol})$ was added dropwise. After $10 \mathrm{~min}$ benzyl bromide (1.45

\footnotetext{
${ }^{1}$ Armarego, W. L. F.; Perrin, D. D. Purification of Laboratory Chemicals; 4th Ed.; Oxford: Butterworth-Heinemann, 1996.

${ }^{2}$ See footnote 50 in: Gao, Y.; Hanson, R.M.; Klunder, J.M.; Ko, S.Y.; Masamune, H.; Sharpless, K.B. J. Am. Chem. Soc. 1987, 109, 5765.

${ }^{3}$ Tamao, K.; Kobayashi, K.; Ito, Y. J. Am. Chem. Soc. 1988, 110, 1286.

${ }^{4}$ Magnus, P.; Exon C.; Albaugh-Robertson, P.. Tetrahedron 1985, 41, 5861.
} 
$\mathrm{mL}, 12 \mathrm{mmol}$ ) was added dropwise, the reaction mixture was stirred for $1 \mathrm{~h}$, and poured into a solution of $\mathrm{NH}_{4} \mathrm{Cl}(25 \mathrm{ml}$, sat'd). The resulting mixture was extracted with hexanes $(3 \times 30 \mathrm{~mL})$, and the combined organic layers were washed with water $(2 \times 20 \mathrm{~mL})$ and $\mathrm{Na}_{2} \mathrm{SO}_{3}(20 \mathrm{~mL}, 10 \%)$, dried $\left(\mathrm{MgSO}_{4}\right)$, filtered (Celite), and concentrated in vacuo. Purification of the residue by flash chromatography on $\mathrm{SiO}_{2}$ (elution gradient: hexanes to $2.5 \% \mathrm{EtOAc} /$ hexanes) provided the title compound as a clear, colorless liquid (1.39 g, 64\%).

$\mathrm{R}_{f} 0.36$ (2.5\% EtOAc/hexanes); IR (thin film) v 3300, 3069, 3031, $1072739,697 \mathrm{~cm}^{-1}$; ${ }^{1} \mathrm{H}$ NMR (300 $\left.\mathrm{MHz}, \mathrm{CDCl}_{3}\right) \delta$ 7.42-7.27 (m, 5H), $5.78(\mathrm{ddd}, \mathrm{J}=7.4,10.3,17.4 \mathrm{~Hz}, 1 \mathrm{H}), 5.35-5.31(\mathrm{~m}, 1 \mathrm{H}), 5.30-5.27$ $(\mathrm{m}, 1 \mathrm{H}), 4.64(\mathrm{~d}, \mathrm{~J}=11.7 \mathrm{~Hz}, 1 \mathrm{H}), 4.40(\mathrm{~d}, \mathrm{~J}=11.7 \mathrm{~Hz}, 1 \mathrm{H}), 3.95(\mathrm{ddd}, \mathrm{J}=5.4,7.4,7.7 \mathrm{~Hz}, 1 \mathrm{H})$, 2.44-2.25 (m, 2H), $1.96(\mathrm{t}, \mathrm{J}=2.8 \mathrm{~Hz}, 1 \mathrm{H}), 1.95-1.70(\mathrm{~m}, 2 \mathrm{H}) ;{ }^{13} \mathrm{C} \mathrm{NMR}\left(75 \mathrm{MHz}, \mathrm{CDCl}_{3}\right) \delta 138.5$, 138.2, 128.3, 127.7, 127.5, 117.6, 84.0, 79.0, 70.3, 68.4, 34.2, 14.6; HRMS m/z 201.12721 (calcd for $\left.\mathrm{C}_{14} \mathrm{H}_{17} \mathrm{O}[\mathrm{M}+\mathrm{H}]^{+}, 201.12794\right)$

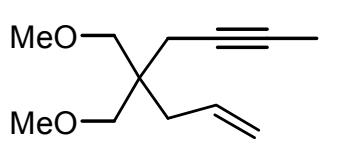

4,4-Bis(methoxymethyl)oct-1-en-6-yne (1b).

To a $0{ }^{\circ} \mathrm{C}$ THF $(30 \mathrm{~mL})$ solution of 4,4-bis(methoxymethyl)hept-1-en-6-yne (5.40 g, $30 \mathrm{mmol})$ was added $n$-BuLi $(13 \mathrm{~mL}, 2.53 \mathrm{M}, 33 \mathrm{mmol})$ dropwise. After $30 \mathrm{~min}$, MeI $(2.3 \mathrm{~mL}, 36 \mathrm{mmol})$ was added dropwise to the solution, the reaction mixture was stirred an additional $\mathrm{h}$, and then allowed to warm to $\mathrm{rt}$. After stirring $10 \mathrm{~h}$ at $\mathrm{rt}$, a solution of $\mathrm{NH}_{4} \mathrm{Cl}(20 \mathrm{~mL}$, sat'd) was added and the layers were separated. The aqueous phase was extracted with ether $(3 \times 30 \mathrm{~mL})$ and the combined layers were washed with $\mathrm{Na}_{2} \mathrm{~S}_{2} \mathrm{O}_{3}(20 \mathrm{~mL}, 10 \%)$ brine $(50 \mathrm{~mL})$, dried $\left(\mathrm{MgSO}_{4}\right)$, filtered (Celite), and concentrated in vacuo. The resulting liquid was purified by vacuum distillation $\left(62-65{ }^{\circ} \mathrm{C}, 0.25 \mathrm{~mm} \mathrm{Hg}\right)$ to give the title compound as a clear, colorless oil $(5.15,89 \%)$.

$\mathrm{R}_{f} 0.33$ (5\% EtOAc/hexanes); IR (thin film) v 3075, 1639, $1109 \mathrm{~cm}^{-1} ;{ }^{1} \mathrm{H}$ NMR $\left(400 \mathrm{MHz}, \mathrm{CDCl}_{3}\right) \delta$ $5.77(\mathrm{ddt}, \mathrm{J}=9.9,17.4,7.5 \mathrm{~Hz}, 1 \mathrm{H}), 5.10-5.01(\mathrm{~m}, 2 \mathrm{H}), 3.30(\mathrm{~s}, 6 \mathrm{H}), 3.22(\mathrm{~d}, \mathrm{~J}=13.0 \mathrm{~Hz}, 2 \mathrm{H}), 3.19(\mathrm{~d}$, $\mathrm{J}=13.0 \mathrm{~Hz}, 2 \mathrm{H}), 2.14-2.08(\mathrm{~m}, 4 \mathrm{H}), 1.77(\mathrm{t}, \mathrm{J}=2.6 \mathrm{~Hz}) ;{ }^{13} \mathrm{C} \mathrm{NMR}\left(100 \mathrm{MHz}, \mathrm{CDCl}_{3}\right) \delta 134.1,117.7$, 77.2, 75.5, 74.4, 59.2, 41.8, 36.2, 22.2, 3.5; HRMS $m / z 197.15423$ (calcd for $\mathrm{C}_{12} \mathrm{H}_{21} \mathrm{O}_{2}[\mathrm{M}+\mathrm{H}]^{+}$, 197.15416).

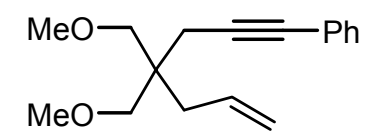

\section{4,4-Bis(methoxymethyl)-7-phenylhept-1-en-6-yne (entry 1).}

$\mathrm{Pd}\left(\mathrm{PPh}_{3}\right)_{2} \mathrm{Cl}_{2}(0.51 \mathrm{~g}, 0.73 \mathrm{mmol})$ and $\mathrm{CuI}(0.28 \mathrm{~g}, 1.5 \mathrm{mmol})$ were added to degassed $\mathrm{NEt}_{3}(24 \mathrm{~mL}, 173 \mathrm{mmol})$ at $\mathrm{rt}$. To the resulting mixture was added 4,4-bis(methoxymethyl)hept-1-en-6-yne (4.37 g, $24 \mathrm{mmol})$. After $30 \mathrm{~min}$, THF (20 mL) was added and the reaction mixture was stirred for $3 \mathrm{~h}$. Volatiles were removed in vacuo, and the remaining residue was triturated with $\mathrm{Et}_{2} \mathrm{O}(5 \times 50 \mathrm{~mL})$ and solids were filtered by passage through a $\mathrm{SiO}_{2}$ plug using $\mathrm{Et}_{2} \mathrm{O}$ for elution. Concentration of the filtrate and purification of the resulting liquid by flash chromatography on $\mathrm{SiO}_{2}$ provided the title compound as a light yellow liquid (5.88 g, 95\%).

$\mathrm{R}_{f} 0.32$ (2.5\% EtOAc/hexanes); IR (thin film) v 3074, 1110, 756, $691 \mathrm{~cm}^{-1} ;{ }^{1} \mathrm{H}$ NMR (400 MHz, $\left.\mathrm{CDCl}_{3}\right) \delta 7.43-7.38(\mathrm{~m}, 2 \mathrm{H}), 7.32-7.25(\mathrm{~m}, 3 \mathrm{H}), 5.85(\mathrm{ddt}, \mathrm{J}=7.5,9.9,17.1 \mathrm{~Hz}, 1 \mathrm{H}), 5.17-5.08(\mathrm{~m}, 2 \mathrm{H})$,

\footnotetext{
${ }^{5}$ Adrio, J.; Rivero, M. R.; Carretero, J. C. Chem. Eur. J. 2001, 7, 2435.
} 
$3.36(\mathrm{~s}, 6 \mathrm{H}), 3.33(\mathrm{~d}, \mathrm{~J}=13.0 \mathrm{~Hz}, 2 \mathrm{H}), 3.31(\mathrm{~d}, \mathrm{~J}=13.0 \mathrm{~Hz}, 2 \mathrm{H}), 2.42(\mathrm{~s}, 2 \mathrm{H}), 2.24(\mathrm{~d}, \mathrm{~J}=7.5 \mathrm{~Hz}, 2 \mathrm{H})$;

${ }^{13} \mathrm{C}$ NMR $\left(100 \mathrm{MHz}, \mathrm{CDCl}_{3}\right) \delta 133.9,131.5,128.2,127.5,124.0,118.1,87.0,82.5,74.5,59.3,42.2$, 36.4, 22.9; HRMS $m / z 259.16990$ (calcd for $\mathrm{C}_{17} \mathrm{H}_{23} \mathrm{O}_{2}[\mathrm{M}+\mathrm{H}]^{+}, 259.16981$ ).

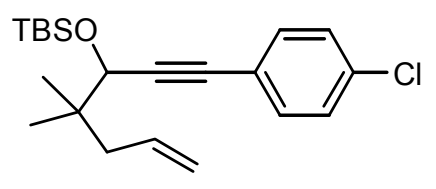

\section{5-tert-Butyldimethylsiloxy-7-(4-chlorophenyl)-4,4-dimethylhept-1-en- 6-yne (entry 4).}

A THF $(20 \mathrm{~mL})$ solution of $p$-chlorophenylacetylene $(2.06 \mathrm{~g}, 15.1 \mathrm{mmol})$ was cooled to $0{ }^{\circ} \mathrm{C}$ and $n$-BuLi $(5.9 \mathrm{~mL}, 2.71 \mathrm{M}, 16 \mathrm{mmol})$ was added dropwise. The acetylide solution was added dropwise to a $0{ }^{\circ} \mathrm{C}$ THF $(15$

$\mathrm{mL})$ solution of 4,4-dimethylpent-1-en-5-al6 $(2.04 \mathrm{~g}, 18.2 \mathrm{mmol})$ via cannula. After $1 \mathrm{~h}$ the ice bath was removed and the reaction mixture was stirred $2 \mathrm{~h}$ at rt. Following cannular addition of a solution of $(t-$ $\mathrm{Bu}) \mathrm{Me}_{2} \mathrm{SiCl}$ in $\mathrm{THF}(10 \mathrm{~mL})$, the reaction mixture was heated at reflux for $11 \mathrm{~h}$. After cooling to rt, $\mathrm{NH}_{4} \mathrm{Cl}(20 \mathrm{~mL}$, sat'd) was added and the mixture was stirred for $5 \mathrm{~min}$. The layers were separated and the aqueous layer was extracted with EtOAc $(3 \times 25 \mathrm{~mL})$. The combined extracts were washed with water $(20 \mathrm{~mL})$ and brine $(20 \mathrm{~mL})$, dried $\left(\mathrm{MgSO}_{4}\right)$, filtered (Celite), and concentrated in vacuo. Vacuum distillation $\left(162-165{ }^{\circ} \mathrm{C}, 0.25 \mathrm{~mm} \mathrm{Hg}\right.$ ) of the liquid provided the title compound as a clear, colorless liquid $(3.89 \mathrm{~g}, 71 \%)$.

$\mathrm{R}_{f} 0.65$ (hexanes); IR (thin film) $v 3076,1640,1257,1091,837 \mathrm{~cm}^{-1} ;{ }^{1} \mathrm{H}$ NMR (300 MHz, $\left.\mathrm{CDCl}_{3}\right) \delta$ 7.39-7.27 (m, 4H), 5.88 (dddd, J = 7.4, 7.4, 9.2, $17.9 \mathrm{~Hz}, 1 \mathrm{H}), 5.13-5.04(\mathrm{~m}, 2 \mathrm{H}), 4.26(\mathrm{~s}, 1 \mathrm{H}), 2.27-2.12$ $(\mathrm{m}, 2 \mathrm{H}), 1.01(\mathrm{~s}, 3 \mathrm{H}), 1.00(\mathrm{~s}, 3 \mathrm{H}), 0.97(\mathrm{~s}, 9 \mathrm{H}), 0.21(\mathrm{~s}, 3 \mathrm{H}), 0.15(\mathrm{~s}, 3 \mathrm{H}),{ }^{13} \mathrm{C}$ NMR $\left(75 \mathrm{MHz}, \mathrm{CDCl}_{3}\right)$ $\delta$ 135.2, 134.0, 132.6, 128.6, 121.8, 117.3, 90.9, 84.4, 70.9, 42.7, 39.5, 25.8, 22.8, 22.7, 18.2, -4.2, -5.2; HRMS $m / z 363.19127$ (calcd for $\mathrm{C}_{21} \mathrm{H}_{32} \mathrm{OSiCl}[\mathrm{M}+\mathrm{H}]^{+}, 363.19110$ ).

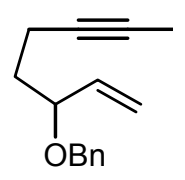

\section{3-Benzyloxyoct-1-en-6-yne (entry 6).}

To a $0{ }^{\circ} \mathrm{C}$ THF $(10 \mathrm{~mL})$ solution of 3-benzyloxyhept-1-en-6-yne $(1.34 \mathrm{~g}, 6.7 \mathrm{mmol}) n$ BuLi $(3.1 \mathrm{~mL}, 2.56 \mathrm{M}, 8.0 \mathrm{mmol})$ was added dropwise. After $30 \mathrm{~min}$, MeI (0.63 mL, 10 $\mathrm{mmol}$ ) was added and the reaction mixture was stirred an additional $1 \mathrm{~h}$ and then poured into a stirred solution of $\mathrm{NH}_{4} \mathrm{Cl}(30 \mathrm{ml}$, sat'd). The layers were separated and the aqueous phase was extracted with EtOAc $(3 \times 30 \mathrm{~mL})$. The combined extracts were washed with $\mathrm{Na}_{2} \mathrm{SO}_{3}(20 \mathrm{~mL}, 10 \%)$ and brine $(30 \mathrm{~mL})$, dried $\left(\mathrm{MgSO}_{4}\right)$, filtered (Celite) and concentrated in vacuo. The resulting oil was purified by flash chromatography on $\mathrm{SiO}_{2}$ (elution gradient: hexanes to $2.5 \% \mathrm{EtOAc/hexanes)} \mathrm{provided}$ the title compound as a clear, colorless liquid $(1.22 \mathrm{~g}, 85 \%)$.

$\mathrm{R}_{f} 0.39$ (2.5\% EtOAc/hexanes); IR (thin film) v 3031, 1070, 736, $698 \mathrm{~cm}^{-1} ;{ }^{1} \mathrm{H}$ NMR (400 MHz, $\left.\mathrm{CDCl}_{3}\right) \delta$ 7.36-7.21 (m, 5H), 5.78-5.65 (m, 1H), 5.28-5.18 (m, 2H), $4.57(\mathrm{dd}, \mathrm{J}=2.1,12.0 \mathrm{~Hz}, 1 \mathrm{H}), 4.35$ (dd, J = 1.7, $12.0 \mathrm{~Hz}, 1 \mathrm{H}), 3.87$ (ddd, $\mathrm{J}=6.2,6.2,6.2 \mathrm{~Hz}, 1 \mathrm{H}), 2.30-2.13(\mathrm{~m}, 2 \mathrm{H}), 1.85-1.58(\mathrm{~m}, 2 \mathrm{H})$, $1.74(\mathrm{t}, \mathrm{J}=2.4 \mathrm{~Hz}, 3 \mathrm{H}) ;{ }^{13} \mathrm{C} \mathrm{NMR}\left(100 \mathrm{MHz}, \mathrm{CDCl}_{3}\right) \delta 138.6,138.4,128.3,127.7,127.4,117.4,79.1$, 78.6, 45.7, 40.2, 13.7, 14.9, 3.4; HRMS $m / z 215.14298$ (calcd for $\mathrm{C}_{15} \mathrm{H}_{19} \mathrm{O}[\mathrm{M}+\mathrm{H}]^{+}, 215.14359$ ).

${ }^{6}$ Magnus, P. D.; Nobbs, M. S. Syn. Commun. 1980, 10, 273. 


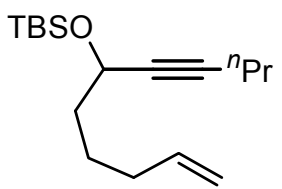

6-tert-Butyldimethylsiloxyundec-1-en-7-yne (entry 7).

A solution of 1-hexen-6-al in $\mathrm{CH}_{2} \mathrm{Cl}_{2}(200 \mathrm{~mL})$ and $\mathrm{Et}_{2} \mathrm{O}(200 \mathrm{~mL})$ was prepared by PCC oxidation of 1-hexen-6-ol $(3.6 \mathrm{~mL}, 30 \mathrm{mmol}){ }^{7}$ To a $0{ }^{\circ} \mathrm{C}$ solution of 1 pentyne in $\mathrm{Et}_{2} \mathrm{O}(50 \mathrm{~mL})$ was added $n$-BuLi $(16 \mathrm{~mL}, 2.71 \mathrm{M}, 43 \mathrm{mmol})$ dropwise. The resulting acetylide solution was added by cannula to the solution of 1-hexen-

6-al at $-78{ }^{\circ} \mathrm{C}$. After $2 \mathrm{~h}$ the cooling bath was removed and the reaction mixture was stirred $10 \mathrm{~h}$ at $\mathrm{rt}$. A solution of $\mathrm{NH}_{4} \mathrm{Cl}(60 \mathrm{~mL}$, sat'd) was added and the layers were separated. The aqueous phase was extracted with $\mathrm{Et}_{2} \mathrm{O}(3 \times 50 \mathrm{~mL})$, and the combined organics were washed with brine $(50 \mathrm{~mL})$, dried $\left(\mathrm{MgSO}_{4}\right)$, filtered and concentrated. The crude alcohol $(1.67 \mathrm{~g}, \sim 10 \mathrm{mmol})$ was taken up in DMF (2 $\mathrm{mL})$ and added to a slurry of imidazole $(1.89 \mathrm{~g}, 28 \mathrm{mmol})$ and $(t-\mathrm{Bu}) \mathrm{Me}_{2} \mathrm{SiCl}(1.84 \mathrm{~g}, 11.1 \mathrm{mmol})$ at $\mathrm{rt}$. After $6 \mathrm{~h}$ the reaction mixture was poured into a stirred solution of $\mathrm{NaHCO}_{3}(30 \mathrm{~mL}$, sat'd). The mixture was extracted with hexanes $(3 \times 30 \mathrm{~mL})$ and the combined extracts were washed with water $(2 \mathrm{x}$ $20 \mathrm{~mL}$ ), dried $\left(\mathrm{MgSO}_{4}\right)$, filtered (Celite), and concentrated in vacuo. The residue was purified by flash chromatography on $\mathrm{SiO}_{2}$ (elution gradient: hexanes to $1 \% \mathrm{EtOAc} /$ hexanes) to give the title compound as a colorless liquid $(2.8 \mathrm{~g}, 33 \%)$.

$\mathrm{R}_{f} 0.26$ (hexanes); IR (thin film) v 3078, 1641, 1253, $1089 \mathrm{~cm}^{-1} ;{ }^{1} \mathrm{H} \mathrm{NMR}\left(300 \mathrm{MHz}, \mathrm{CDCl}_{3}\right) \delta 5.83$ (dddd, J = 6.7, 6.7, 10.3, 16.9 Hz, 1 H), 5.08-4.94 (m, 2H), 4.36 (ddd, J 2.1, 2.1, 6.4, 6.4 Hz, 1H), 2.19 (app dt, J =2.1, 6.9 Hz, 2H), 2.09 (app q, J = 6.9 Hz, 2H), 1.72-1.61 (m, 2H), 1.60-1.47 (m, 4H), 0.99 $(\mathrm{s}, 9 \mathrm{H}), 0.14(\mathrm{~s}, 3 \mathrm{H}), 0.13(\mathrm{~s}, 3 \mathrm{H}) ;{ }^{13} \mathrm{C} \mathrm{NMR}\left(75 \mathrm{MHz}, \mathrm{CDCl}_{3}\right) \delta 138.8,114.4,84.3,82.0,63.0,38.4$, 33.4, 25.8, 24.6, 22.1, 20.7, 18.3, 13.5, -4.5, -5.0; HRMS $m / z 281.22940$ (calcd for $\mathrm{C}_{17} \mathrm{H}_{33} \mathrm{OSi}[\mathrm{M}+\mathrm{H}]^{+}$, 281.30007).

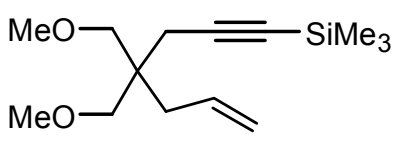

\section{4,4-Bis(methoxymethyl)-7-trimethylsilylhept-1-en-6-yne (entry 9).} To a $0{ }^{\circ} \mathrm{C}$ THF $(30 \mathrm{~mL})$ solution of 4,4-bis(methoxymethyl)hept-1-en-6yne $(5.40 \mathrm{~g}, 30 \mathrm{mmol})$ was added $n$-BuLi $(13 \mathrm{~mL}, 2.53 \mathrm{M}, 33 \mathrm{mmol})$ dropwise. After $30 \mathrm{~min}, \mathrm{Me}_{3} \mathrm{SiCl}(4.5 \mathrm{~mL}, 36 \mathrm{mmol})$ was added dropwise to the canary yellow solution, the reaction mixture was stirred an additional $h$, and then allowed to warm to rt. After stirring $10 \mathrm{~h}$ at $\mathrm{rt}$, a solution of $\mathrm{NH}_{4} \mathrm{Cl}(20 \mathrm{~mL}$, sat'd) was added and the layers were separated. The aqueous phase was extracted with EtOAc $(3 \times 30 \mathrm{~mL})$ and the combined layers were washed with brine $(50 \mathrm{~mL})$, dried $\left(\mathrm{MgSO}_{4}\right)$, filtered (Celite), and concentrated in vacuo. The resulting liquid was purified by vacuum distillation $\left(80-83{ }^{\circ} \mathrm{C}, 0.25 \mathrm{~mm} \mathrm{Hg}\right)$ to give the title compound as a clear, colorless oil $(6.48 \mathrm{~g}, 86 \%)$.

$\mathrm{R}_{f} 0.35$ (2.5\% EtOAc/hexanes); IR (thin film) v 3076, 2174, 1653, 1250, $1110 \mathrm{~cm}^{-1}$; ${ }^{1} \mathrm{H}$ NMR (400 $\left.\mathrm{MHz}_{\mathrm{CDCl}} \mathrm{CD}\right) .77(\mathrm{ddt}, \mathrm{J}=9.9,17.1,7.5 \mathrm{~Hz}, 1 \mathrm{H}), 5.10-5.01(\mathrm{~m}, 2 \mathrm{H}), 3.30(\mathrm{~s}, 6 \mathrm{H}), 3.22(\mathrm{~d}, \mathrm{~J}=12.3$ $\mathrm{Hz}, 2 \mathrm{H}), 3.20(\mathrm{~d}, \mathrm{~J}=12.3 \mathrm{~Hz}), 2.18(\mathrm{~s}, 2 \mathrm{H}), 2.13(\mathrm{~d}, \mathrm{~J}=7.5 \mathrm{~Hz}), 0.13(\mathrm{~s}, 9 \mathrm{H}) ;{ }^{13} \mathrm{C} \mathrm{NMR}(100 \mathrm{MHz}$, $\mathrm{CDCl}_{3}$ ) $\delta 133.9,118.0,104.2,86.6,74.3,59.3,41.9,36.2,23.3,0.1$; HRMS $\mathrm{m} / z$ 255.17828 (calcd for $\left.\mathrm{C}_{14} \mathrm{H}_{27} \mathrm{O}_{2} \mathrm{Si}[\mathrm{M}+\mathrm{H}]^{+}, 255.17803\right)$.

\footnotetext{
${ }^{7}$ Spino, C.; Barriault, N. J. Org. Chem. 1999, 64, 5292.
} 


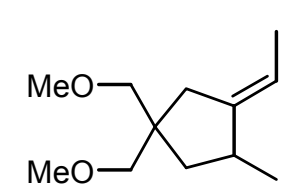

\section{E-Ethylidene-1,1-bis(methoxymethyl)-4-methylcyclopentane (4b).}

To a solution of $\mathrm{Cp}_{2} \mathrm{ZrCl}_{2}(98 \mathrm{mg}, 0.34 \mathrm{mmol})$ in THF $(3 \mathrm{~mL})$ cooled to $-78{ }^{\circ} \mathrm{C}$, was added $n$-BuLi $(0.26 \mathrm{~mL}, 2.56 \mathrm{M}, 0.68 \mathrm{mmol})$ and the dry ice bath was removed. The reaction mixture was allowed to warm until homogenous $\left(\sim 0{ }^{\circ} \mathrm{C}\right)$, at which point the mixture was cooled to $-78^{\circ} \mathrm{C}$. A solution of the enyne $(63 \mathrm{mg}$, $0.32 \mathrm{mmol})$ in THF $(1.5 \mathrm{~mL})$ was added via cannula, and the dry ice bath was replaced with an ice bath. After $1 \mathrm{~h}$ the ice bath was removed and the reaction mixture was allowed to warm to rt. After $12 \mathrm{~h}, \mathrm{HCl}$ $(0.5 \mathrm{~mL}, 3 \mathrm{M}, 1.5 \mathrm{mmol})$ was added and the mixture was stirred for $10 \mathrm{~min}$. The layers were separated and the aqueous phase was extracted with hexanes $(3 \times 15 \mathrm{~mL})$. The combined extracts were washed with $\mathrm{NaHCO}_{3}$ (sat'd) and brine, dried $\left(\mathrm{MgSO}_{4}\right)$, filtered and concentrated. Purification by flash chromatography on $\mathrm{SiO}_{2}$ (elution gradient: hexanes to $2.5 \%$ EtOAc/hexanes) provided the title compound as a colorless oil (48 $\mathrm{mg}, 75 \%$ ).

$\mathrm{R}_{f} 0.23$ (2.5\% EtOAc/hexanes); IR (thin film) $v 3035,1198,1111 \mathrm{~cm}^{-1} ;{ }^{1} \mathrm{H}$ NMR $\left(300 \mathrm{MHz}, \mathrm{CDCl}_{3}\right) \delta$ 5.18-5.08 (m, 1H), $3.31(\mathrm{~s}, 3 \mathrm{H}), 3.30(\mathrm{~s}, 3 \mathrm{H}), 3.25(\mathrm{~d}, \mathrm{~J}=8.7 \mathrm{~Hz}, 1 \mathrm{H}), 3.20(\mathrm{~d}, \mathrm{~J}=8.7 \mathrm{~Hz}, 1 \mathrm{H}), 3.19$ (s, 2H), 2.53-2.38 (m, 1H), 2.12 (s, 2H), $1.82(\mathrm{dd}, \mathrm{J}=7.7,12.8 \mathrm{~Hz}, 1 \mathrm{H}), 1.57-1.51(\mathrm{~m}, 3 \mathrm{H}), 1.04-0.94(\mathrm{~m}, 1$ $\mathrm{H}), 1.00(\mathrm{~d}, \mathrm{~J}=6.7 \mathrm{~Hz}, 3 \mathrm{H}) ;{ }^{13} \mathrm{C}$ NMR $\left(75 \mathrm{MHz}, \mathrm{CDCl}_{3}\right) \delta 146.9,114.0,77.8,75.4,59.3,45.5,40.6$, 36.4, 35.7, 31.6, 18.6, 14.4; HRMS m/z 199.16999 (calcd for $\mathrm{C}_{12} \mathrm{H}_{23} \mathrm{O}_{2}[\mathrm{M}+\mathrm{H}]^{+}$, 199.16981).

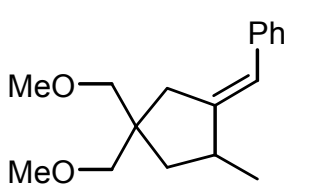

\section{E-Benzylidene-1,1-bis(methoxymethyl)-4-methylcyclopentane (entry 1).} The title compound was prepared from the corresponding enyne $(129 \mathrm{mg}, 0.50$ $\mathrm{mmol}$ ) according to the procedure described for $\mathbf{4 b}$ (light yellow oil, $116 \mathrm{mg}$, $89 \%)$.

$\mathrm{R}_{f} 0.35$ (2.5\% EtOAc/hexanes); IR (thin film) v 3022, 1198, 1107, 747, $696 \mathrm{~cm}^{-1} ;{ }^{1} \mathrm{H}$ NMR $(400 \mathrm{MHz}$, $\left.\mathrm{CDCl}_{3}\right) \delta$ 7.34-7.30 (m, 4H), 7.21-7.14 (m, 1H), $6.22(\mathrm{ddd}, \mathrm{J}=2.4,2.4,2.4 \mathrm{~Hz}, 1 \mathrm{H}), 3.34(\mathrm{~s}, 6 \mathrm{H}), 3.31$ $(\mathrm{d}, \mathrm{J}=8.6 \mathrm{~Hz}, 1 \mathrm{H}), 3.28(\mathrm{~d}, \mathrm{~J}=8.6 \mathrm{~Hz}, 1 \mathrm{H}), 3.27(\mathrm{~s}, 2 \mathrm{H}), 2.84-2.71(\mathrm{~m} \mathrm{1H}), 2.58(\mathrm{~d}, \mathrm{~J}=17.4 \mathrm{~Hz}, 1 \mathrm{H})$, $2.52(\mathrm{dd}, \mathrm{J}=2.4,17.4 \mathrm{~Hz}, 1 \mathrm{H}), 1.93(\mathrm{dd}, \mathrm{J}=7.9,12.7 \mathrm{~Hz}, 1 \mathrm{H}) 1.21(\mathrm{~d}, \mathrm{~J}=6.5 \mathrm{~Hz}, 3 \mathrm{H}), 1.14$ (dd, J = $11.3,12.7 \mathrm{~Hz}, 1 \mathrm{H}) ;{ }^{13} \mathrm{C}$ NMR $\left(100 \mathrm{MHz}, \mathrm{CDCl}_{3}\right) \delta 149.7,138.4,128.2,128.1,125.7,120.7,77.5,75.4$, 59.2, 59.2, 46.5, 39.5, 38.4, 37.8, 18.9; HRMS $m / z 260.17678$ (calcd for $\mathrm{C}_{17} \mathrm{H}_{24} \mathrm{O}_{2}[\mathrm{M}]^{+}$, 260.177630).

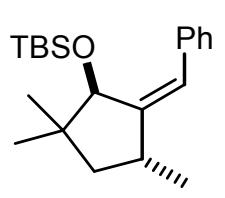

\section{trans-2Z-Benzylidene-1-tert-butyldimethylsiloxy-3,5,5-trimethylcyclopentane (entry 3). ${ }^{8}$}

The title compound was prepared from the corresponding enyne $(164 \mathrm{mg}, 0.50 \mathrm{mmol})$ according to the procedure described for $\mathbf{4 b}$ (colorless liquid, $122 \mathrm{mg}, 74 \%$ ).

$\mathrm{R}_{f} 0.74$ (hexanes); IR (thin film) v 3024, 1252, 1068, 737, $698 \mathrm{~cm}^{-1} ;{ }^{1} \mathrm{H}$ NMR $\left(400 \mathrm{MHz}, \mathrm{CDCl}_{3}\right) \delta$ 7.35-7.19 (m, 5H), $6.30(\mathrm{~d}, \mathrm{~J}=3.6 \mathrm{~Hz}, 1 \mathrm{H}), 4.48(\mathrm{~s}, 1 \mathrm{H}), 3.02-2.91(\mathrm{~m}, 1 \mathrm{H}), 2.15(\mathrm{dd}, \mathrm{J}=12.0,12.0 \mathrm{~Hz}$, $1 \mathrm{H}), 1.20(\mathrm{~d}, \mathrm{~J}=8.8 \mathrm{~Hz}, 3 \mathrm{H}), 1.10(\mathrm{dd}, \mathrm{J}=8.0,16.0 \mathrm{~Hz}, 1 \mathrm{H}), 1.07(\mathrm{~s}, 3 \mathrm{H}), 0.98(\mathrm{~s}, 3 \mathrm{H}), 0.80(\mathrm{~s}, 9 \mathrm{H}),-$ $0.30(\mathrm{~s}, 3 \mathrm{H}),-0.32(\mathrm{~s}, 3 \mathrm{H}) ;{ }^{13} \mathrm{C} \mathrm{NMR}\left(100 \mathrm{MHz}, \mathrm{CDCl}_{3}\right) \delta 154.1,138.8,128.5,128.1,126.2,121.9$, 79.1, 45.4, 41.6, 34.5, 28.1, 25.8, 23.7, 20.0, 18.2, -4.9, -5.4; HRMS $\mathrm{m} / \mathrm{z} 330.23738$ (calcd for $\left.\mathrm{C}_{21} \mathrm{H}_{34} \mathrm{OSi}[\mathrm{M}]^{+}, 330.23789\right)$.

\footnotetext{
${ }^{8}$ This compound was previously reported (Oh, C. H.; Jung, H. H.; Sung, H. R.; Kim, J. D. Tetrahedron 2001, 57, 1723), but $1 \mathrm{H}$ NMR and 13C NMR data were inconsistent with the current work.
} 


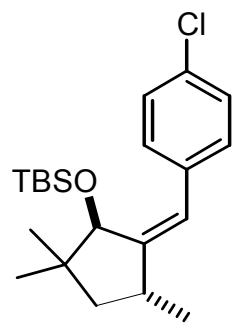

trans-1-tert-Butyldimethylsiloxy-2Z-(4-chlorophenyl)vinylidene-3,5,5trimethylcyclopentane (entry 4).

The title compound was prepared from the corresponding enyne (360 mg, 0.99 $\mathrm{mmol}$ ) according to the procedure described for $\mathbf{4 b}$ (colorless liquid, $221 \mathrm{mg}, 61 \%$ ).

$\mathrm{R}_{f} 0.70$ (hexanes); IR (thin film) $v 1254,1073,836 \mathrm{~cm}^{-1} ;{ }^{1} \mathrm{H}$ NMR $(400 \mathrm{MHz}$, $\left.\mathrm{CDCl}_{3}\right) \delta 7.29-7.24(\mathrm{~m}, 2 \mathrm{H}), 7.18-7.13(\mathrm{~m}, 2 \mathrm{H}), 6.19(\mathrm{~d}, \mathrm{~J}=2.7 \mathrm{~Hz}, 1 \mathrm{H}), 4.36(\mathrm{~s}$, $1 \mathrm{H}), 2.98-2.87(\mathrm{~m}, 1 \mathrm{H}), 2.10(\mathrm{dd}, \mathrm{J}=12.0,12.0 \mathrm{~Hz}, 1 \mathrm{H}), 1.15(\mathrm{~d}, \mathrm{~J}=7.2 \mathrm{~Hz}, 3 \mathrm{H})$, $1.06(\mathrm{dd}, \mathrm{J}=5.1,12.7 \mathrm{~Hz}, 1 \mathrm{H}), 1.03(\mathrm{~s}, 3 \mathrm{H}), 0.92(\mathrm{~s}, 3 \mathrm{H}), 0.77(\mathrm{~s}, 9 \mathrm{H}),-0.30(\mathrm{~s}, 3 \mathrm{H}),-0.35(\mathrm{~s}, 3 \mathrm{H}) ;{ }^{13} \mathrm{C}$ NMR $\left(100 \mathrm{MHz}, \mathrm{CDCl}_{3}\right) \delta 155.0,137.2,131.9,129.7,128.3,120.7,79.1,45.2,41.6,34.6,28.0,35.7$, 23.6, 19.9, 18.2, -4.8, -5.2; HRMS m/z 364.19800 (calcd for $\mathrm{C}_{21} \mathrm{H}_{33} \mathrm{OSiCl}[\mathrm{M}]^{+}, 364.19892$ ).

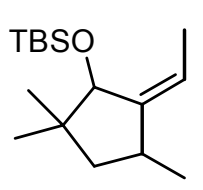

1-tert-Butyldimethylsiloxy-2Z-ethylidene-3,5,5-trimethylcyclopentane (entry 5). The title compound was prepared from the corresponding enyne (133 $\mathrm{mg}, 0.50 \mathrm{mmol})$ according to the procedure described for $\mathbf{4 b}$ as an inseparable mixture of cis/trans isomers (1:1.5 dr) (colorless liquid, $111 \mathrm{mg}, 83 \%)$.

trans: $\mathrm{R}_{f} 0.87$ (hexanes); ${ }^{1} \mathrm{H}$ NMR $\left(400 \mathrm{MHz}, \mathrm{CDCl}_{3}\right) \delta 5.19(\mathrm{dq}, \mathrm{J}=2.4,6.8 \mathrm{~Hz}, 1 \mathrm{H}), 4.05(\mathrm{~s}, 1 \mathrm{H})$, 2.76-2.64 (m, 1H), 1.97 (dd, J = 10.9, $12.3 \mathrm{~Hz}, 1 \mathrm{H}), 1.71(\mathrm{dd}, \mathrm{J}=2.7,6.8 \mathrm{~Hz}, 3 \mathrm{H}), 1.03$ (d, J = 6.8 Hz, $3 \mathrm{H}), 1.00(\mathrm{~s}, 3 \mathrm{H}), 0.95$ (dd, J = 5.5, $12.3 \mathrm{~Hz}, 1 \mathrm{H}), 0.87$ (s, 9H), 0.77 (s, 3H), 0.07 (s, 3H), $0.02(\mathrm{~s}, 3 \mathrm{H})$; ${ }^{13} \mathrm{C}$ NMR $\left(100 \mathrm{MHz}, \mathrm{CDCl}_{3}\right) \delta 150.6,115.5,79.5,45.5,41.2,34.0,27.8,25.9,23.6,19.6,18.3,15.0$, 4.4, -4.5; HRMS $m / z$ 269.22926 (calcd for $\mathrm{C}_{16} \mathrm{H}_{33} \mathrm{OSi}[\mathrm{M}+\mathrm{H}]^{+}, 269.23007$ ).

cis: $\mathrm{R}_{f} 0.87$ (hexanes); ${ }^{1} \mathrm{H}$ NMR $\left(400 \mathrm{MHz}, \mathrm{CDCl}_{3}\right) \delta 5.29$ (ddq, J = 0.7, 1.7, $\left.6.8 \mathrm{~Hz}, 1 \mathrm{H}\right), 3.94(\mathrm{~s}, 1 \mathrm{H})$, 2.52-2.39 (m, 1H), $1.70(\mathrm{dd}, \mathrm{J}=1.7,6.8 \mathrm{~Hz}, 3 \mathrm{H}), 1.52(\mathrm{dd}, \mathrm{J}=8.2,12.0 \mathrm{~Hz}, 1 \mathrm{H}), 1.40(\mathrm{dd}, \mathrm{J}=8.6,12.3$ $\mathrm{Hz}, 1 \mathrm{H}), 1.10(\mathrm{~d}, \mathrm{~J}=7.2 \mathrm{~Hz}, 3 \mathrm{H}), 1.01(\mathrm{~s}, 3 \mathrm{H}), 0.88(\mathrm{~s}, 9 \mathrm{H}), 0.73(\mathrm{~s}, 3 \mathrm{H}), 0.08(\mathrm{~s}, 3 \mathrm{H}), 0.03(\mathrm{~s}, 3 \mathrm{H}) ;{ }^{13} \mathrm{C}$ $\operatorname{NMR}\left(100 \mathrm{MHz}, \mathrm{CDCl}_{3}\right) \delta 150.6,118.8,78.7,44.9,43.0,36.1,25.9,25.4,23.8,23.4,18.3,15.5$, -4.2, 4.6.

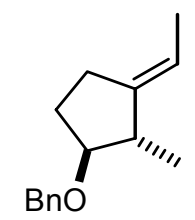

trans-1-Benzyloxy-3E-ethylidene-2-methylcyclopentane (entry 6).

The title compound was prepared from the corresponding enyne (107 $\mathrm{mg}, 0.50 \mathrm{mmol})$ according to the procedure described for $\mathbf{4 b}$ as an inseparable mixture of cis/trans isomers (1:8 dr) (colorless liquid, $89 \mathrm{mg}, 82 \%)$.

$\mathrm{R}_{f} 0.44$ (5\% EtOAc/hexanes); IR (thin film) v 3064, 3031, 1105, 736, $697 \mathrm{~cm}^{-1} ;{ }^{1} \mathrm{H}$ NMR (400 MHz, $\left.\mathrm{CDCl}_{3}\right) \delta 7.40-7.25(\mathrm{~m}, 5 \mathrm{H}), 5.31-5.23(\mathrm{~m}, 1 \mathrm{H}), 4.63(\mathrm{~d}, \mathrm{~J}=2.0 \mathrm{~Hz}, 1 \mathrm{H}), 4.53(\mathrm{~d}, \mathrm{~J}=12.0 \mathrm{~Hz}, 1 \mathrm{H}) 3.45$ $(\mathrm{ddd}, \mathrm{J}=6.6,6.6,6.6 \mathrm{~Hz}, 1 \mathrm{H}), 2.56-2.36(\mathrm{~m}, 2 \mathrm{H}), 2.28-2.13(\mathrm{~m}, 1 \mathrm{H}), 2.09-1.99(\mathrm{~m}, 1 \mathrm{H}), 1.73-1.60(\mathrm{~m}$, $1 \mathrm{H}), 1.60(\mathrm{~d}, \mathrm{~J}=6.8 \mathrm{~Hz}, 3 \mathrm{H}), 1.10(\mathrm{~d}, \mathrm{~J}=6.8 \mathrm{~Hz}, 3 \mathrm{H}) ;{ }^{13} \mathrm{C} \mathrm{NMR}\left(100 \mathrm{MHz}, \mathrm{CDCl}_{3}\right) \delta 144.6,138.9$, 128.3, 127.4, 115.0, 86.1, 71.1, 44.6, 29.1, 25.5, 17.1, 14.0; HRMS $m / z 217.15888$ (calcd for $\mathrm{C}_{15} \mathrm{H}_{21} \mathrm{O}$ $\left.[\mathrm{M}+\mathrm{H}]^{+}, 217.15924\right)$.

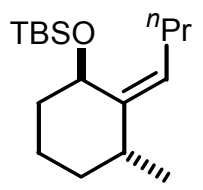

trans-1-tert-Butyldimethylsiloxy-2Z-butylidene-3-methylcyclohexane (entry 7). The title compound was prepared from the corresponding enyne (140 $\mathrm{mg}, 0.50 \mathrm{mmol})$ according to the procedure described for $\mathbf{4 b}$ (colorless liquid, $79 \mathrm{mg}, 56 \%$ ). 
$\mathrm{R}_{f} 0.83$ (hexanes); IR (thin film) v 1254, $1110 \mathrm{~cm}^{-1} ;{ }^{1} \mathrm{H}$ NMR (400 MHz, $\left.\mathrm{CDCl}_{3}\right) \delta 4.95$ (ddd, J = 1.7, 7.2, 7.2 Hz, 1H), $4.75(\mathrm{dd}, \mathrm{J}=2.9,2.9 \mathrm{~Hz}, 1 \mathrm{H}), 2.57-2.45(\mathrm{~m}, 1 \mathrm{H}), 2.03(\operatorname{app~dq}, \mathrm{J}=3.8,4.8,13.3 \mathrm{~Hz})$, 1.84-1.70 (m, 2H), 1.53-1.19 (m, 5H), 0.95-0.87 (m, 6H), $0.88(\mathrm{~s}, 9 \mathrm{H}), 0.04(\mathrm{~s}, 3 \mathrm{H}),-0.03(\mathrm{~s}, 3 \mathrm{H}) ;{ }^{13} \mathrm{C}$ NMR $\left(100 \mathrm{MHz}, \mathrm{CDCl}_{3}\right) \delta 145.4,118.8,66.1,37.6,35.8,32.1,29.1,25.8,23.4,20.4,18.3,18.0,13.9$, 4.7, -4.9; HRMS m/z 283.24485 (calcd for $\mathrm{C}_{17} \mathrm{H}_{35} \mathrm{OSi}[\mathrm{M}+\mathrm{H}]^{+}, 283.24572$ ).

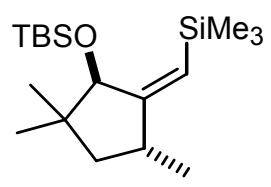

trans-1-tert-Butyldimethylsiloxy-3,5,5-trimethyl-2Ztrimethylsilylvinylidenecyclopentane (entry 8).

The title compound was prepared from the corresponding enyne (162 mg, 0.50 $\mathrm{mmol}$ ) according to the procedure described for $\mathbf{4 b}$ (colorless liquid, $141 \mathrm{mg}$, $86 \%)$.

$\mathrm{R}_{f} 0.87$ (hexanes); IR (thin film) $v 1636,1249,1079 \mathrm{~cm}^{-1} ;{ }^{1} \mathrm{H}$ NMR $\left(400 \mathrm{MHz}, \mathrm{CDCl}_{3}\right) \delta 5.18(\mathrm{~d}, \mathrm{~J}=$ $2.4 \mathrm{~Hz}, 1 \mathrm{H}), 3.90(\mathrm{~s}, 1 \mathrm{H}), 2.86-2.75(\mathrm{~m}, 1 \mathrm{H}), 1.99(\mathrm{dd}, \mathrm{J}=11.8 \mathrm{~Hz}, 1 \mathrm{H}), 1.02(\mathrm{~s}, 3 \mathrm{H}), 1.00(\mathrm{~s}, 3 \mathrm{H}), 0.85$ $(\mathrm{s}, 9 \mathrm{H}), 0.72(\mathrm{~s}, 3 \mathrm{H}), 0.11(\mathrm{~s}, 9 \mathrm{H}), 0.06(\mathrm{~s}, 3 \mathrm{H}), 0.02(\mathrm{~s}, 3 \mathrm{H}) ;{ }^{13} \mathrm{C} \mathrm{NMR}\left(100 \mathrm{MHz}, \mathrm{CDCl}_{3}\right) \delta 167.5$, 118.7, 82.6, 44.7, 40.5, 35.8, 28.0, 25.8, 23.8, 19.1, 18.4, 0.6, -3.8, -4.3; HRMS m/z 327.25316 (calcd for $\left.\mathrm{C}_{18} \mathrm{H}_{38} \mathrm{OSi}_{2}[\mathrm{M}+\mathrm{H}]^{+}, 327.25395\right)$.

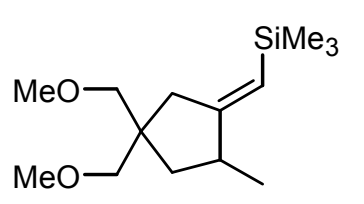

\section{1,1-Bis(methoxymethyl)-4-methyl $3 E$-trimethylsilylvinylidenecyclopentane (entry 9).}

The title compound was prepared from the corresponding enyne ( $247 \mathrm{mg}, 0.97$ mmol) according to the procedure described for $\mathbf{4 b}$ except that $\mathrm{H}_{2} \mathrm{SO}_{4}(1.3 \mathrm{~mL}$, $10 \%$ ) was used for protonation of the zirconocycle (colorless liquid, $236 \mathrm{mg}$,

$95 \%)$.

$\mathrm{R}_{f} 0.33$ (5\% EtOAc/hexanes); IR (thin film) v 1637, 1248, 1110, $\mathrm{cm}^{-1} ;{ }^{1} \mathrm{H}$ NMR (300 MHz, $\left.\mathrm{CDCl}_{3}\right) \delta$ $5.21(\mathrm{ddd}, \mathrm{J}=2.3,2.3,2.3 \mathrm{~Hz}, 1 \mathrm{H}), 3.31(\mathrm{~s}, 3 \mathrm{H}), 3.31(\mathrm{~s}, 3 \mathrm{H}), 3.22(\mathrm{~d}, \mathrm{~J}=11.01 \mathrm{~Hz}, 2 \mathrm{H}), 3.19(\mathrm{~d}, \mathrm{~J}=$ $11.0 \mathrm{~Hz}, 2 \mathrm{H}), 2.54-2.40(\mathrm{~m}, 1 \mathrm{H}), 2.24(\operatorname{app~s}, 2 \mathrm{H}), 1.84(\mathrm{dd}, \mathrm{J}=8.5,13.1 \mathrm{~Hz}, 1 \mathrm{H}), 1.02(\mathrm{~d}, \mathrm{~J}=6.7 \mathrm{~Hz}$, $3 \mathrm{H}), 1.01(\mathrm{dd}, \mathrm{J}=10.8,12.8 \mathrm{~Hz}, 1 \mathrm{H}), 0.06(\mathrm{~s}, 9 \mathrm{H}) ;{ }^{13} \mathrm{C} \mathrm{NMR}\left(75 \mathrm{MHz}, \mathrm{CDCl}_{3}\right) \delta 165.8,117.3,77.4$, 75.6, 59.3, 59.2, 45.6, 39.6, 39.2, 39.0, 18.7, -0.2; HRMS $m / z 256.18354$ (calcd for $\mathrm{C}_{14} \mathrm{H}_{28} \mathrm{O}_{2} \mathrm{Si}[\mathrm{M}]^{+}$, 256.18586).

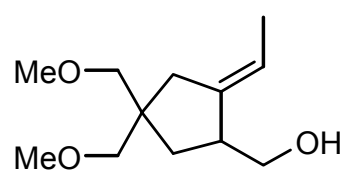

\section{E-Ethylidene-4-hydroxymethyl-1,1-bis(methoxymethyl)cyclopentane}

(3b).

To a solution of $\mathrm{Cp}_{2} \mathrm{ZrCl}_{2}(306 \mathrm{mg}, 1.05 \mathrm{mmol})$ in THF (3 mL) cooled to -78

${ }^{\circ} \mathrm{C}$, was added $n$-BuLi $(0.81 \mathrm{~mL}, 2.71 \mathrm{M}, 2.20 \mathrm{mmol})$ and the dry ice bath was removed. The reaction mixture was allowed to warm until homogenous $(\sim 0$

$\left.{ }^{\circ} \mathrm{C}\right)$, at which point the mixture was cooled to $-78{ }^{\circ} \mathrm{C}$. A solution of the enyne $(0.190 \mathrm{mg}, 0.97 \mathrm{mmol})$ in THF $(1.5 \mathrm{~mL})$ was added via cannula, and the dry ice bath was replaced with an ice bath. After $1 \mathrm{~h}$ the ice bath was removed and the reaction mixture was allowed to warm to rt. Neat $\left({ }^{\mathrm{c}} \mathrm{Hex}\right)_{2} \mathrm{BCl}(0.50$ $\mathrm{mL}, 2.3 \mathrm{mmol}$ ) was added $12 \mathrm{~h}$ later by syringe. After $5 \mathrm{~h}$ the borane was oxidized by successive treatment with aqueous $\mathrm{NaOH}(2 \mathrm{~mL}, 7.5 \mathrm{M}, 15 \mathrm{mmol})$ and $\mathrm{H}_{2} \mathrm{O}_{2}(2 \mathrm{~mL}, 30 \%)$ using a water bath to control the exotherm. The reaction mixture was then heated at $50{ }^{\circ} \mathrm{C}$ for $1 \mathrm{~h}$, allowed to cool, and the layers were separated. The aqueous layer was extracted with EtOAc $(3 \times 20 \mathrm{~mL})$ and the extracts were washed with $\mathrm{Na}_{2} \mathrm{SO}_{3}(2 \times 10 \mathrm{~mL}, 10 \%), \mathrm{NaHCO}_{3}$ (sat'd) and brine, dried $\left(\mathrm{MgSO}_{4}\right)$, filtered and 
concentrated. Purification of the residue by flash chromatography on $\mathrm{SiO}_{2}$ (elution gradient: hexanes to

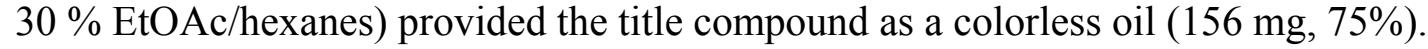

$\mathrm{R}_{f} 0.18$ (30\% EtOAc/hexanes); IR (thin film) v 3386 (br), 1198, $1109 \mathrm{~cm}^{-1} ;{ }^{1} \mathrm{H}$ NMR (400 MHz, $\left.\mathrm{CDCl}_{3}\right) \delta 5.30$ (dddq, J = 2.1, 2.4, 2.4, $\left.6.8 \mathrm{~Hz}, 1 \mathrm{H}\right), 3.58(\mathrm{~d}, \mathrm{~J}=5.5 \mathrm{~Hz}, 2 \mathrm{H}), 3.32(\mathrm{~s}, 3 \mathrm{H}), 3.30(\mathrm{~s}, 3 \mathrm{H})$, $3.30(\mathrm{~d}, \mathrm{~J}=8.9 \mathrm{~Hz}, 1 \mathrm{H}), 3.26(\mathrm{~d}, \mathrm{~J}=8.9 \mathrm{~Hz}, 1 \mathrm{H}), 3.17(\mathrm{~d}, \mathrm{~J}=11.1 \mathrm{~Hz}, 1 \mathrm{H}), 3.15(\mathrm{~d}, \mathrm{~J}=11.1 \mathrm{~Hz}, 1 \mathrm{H})$, 2.72-2.61 (m, 1H), $2.23(\mathrm{~d}, \mathrm{~J}=16.7 \mathrm{~Hz}, 1 \mathrm{H}), 2.07(\mathrm{dd}, \mathrm{J}=2.1,16.7 \mathrm{~Hz}, 1 \mathrm{H}), 1.81(\mathrm{dd}, \mathrm{J}=7.5,13.0 \mathrm{~Hz}$, $1 \mathrm{H}), 1.58(\mathrm{~d}, \mathrm{~J}=6.8 \mathrm{~Hz}, 3 \mathrm{H}), 1.36(\mathrm{dd}, \mathrm{J}=9.2,13.0 \mathrm{~Hz}, 1 \mathrm{H}){ }^{13} \mathrm{C} \mathrm{NMR}\left(100 \mathrm{MHz}, \mathrm{CDCl}_{3}\right) \delta 142.4$, $116.8,77.4,75.3,65.4,59.3,46.1,44.6,35.7,34.4,14.7$; HRMS $m / z 215.16513$ (calcd for $\mathrm{C}_{12} \mathrm{H}_{23} \mathrm{O}_{3}$ $\left.[\mathrm{M}+\mathrm{H}]^{+}, 215.16472\right)$.

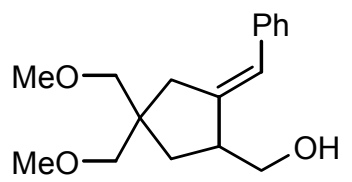

\title{
3E-Benzylidene-4-hydroxymethyl-1,1-bis(methoxymethyl)cyclopentane (entry 1). \\ The title compound was prepared from the corresponding enyne $(256 \mathrm{mg}$, $0.99 \mathrm{mmol}$ ) according to the procedure described for $\mathbf{3 b}$ (colorless oil, 227 $\mathrm{mg}, 83 \%)$.
}

$\mathrm{R}_{f} 0.20$ (25\% EtOAc/hexanes); IR (thin film) v 3417 (br), $1108 \mathrm{~cm}^{-1} ;{ }^{1} \mathrm{H} \mathrm{NMR}\left(400 \mathrm{MHz}, \mathrm{CDCl}_{3}\right) \delta$ 7.35-7.25 (m, 4H), 7.22-7.15 (m, 1H), 6.37 (s, 1H), 5.73 (d, J = 5.8 Hz, 2H), 3.39-3.31 (m, 2H), 3.36 (s, $3 \mathrm{H}), 3.28(\mathrm{~s}, 3 \mathrm{H}), 3.17(\mathrm{~s}, 2 \mathrm{H}), 2.98-2.87(\mathrm{~m}, 1 \mathrm{H}), 2.60(\mathrm{~d}, \mathrm{~J}=17.1 \mathrm{~Hz}, 1 \mathrm{H}), 2.50(\mathrm{~d}, \mathrm{~J}=17.1 \mathrm{~Hz}, 1 \mathrm{H})$, 1.99 (br s, $1 \mathrm{H}), 1.90$ (dd, J = 8.6, $13.0 \mathrm{~Hz}, 1 \mathrm{H}), 1.47$ (dd, J = 8.9, $13.0 \mathrm{~Hz}, 1 \mathrm{H}) ;{ }^{13} \mathrm{C}$ NMR $(100 \mathrm{MHz}$, $\left.\mathrm{CDCl}_{3}\right) \delta 145.2,137.9,128.4,128.1,126.1,123.0,77.1,75.3,65.9,59.2,59.2,47.3,46.6,37.9,33.3$; HRMS $m / z 277.18117$ (calcd for $\mathrm{C}_{17} \mathrm{H}_{25} \mathrm{O}_{3}[\mathrm{M}+\mathrm{H}]^{+}, 277.18037$ ).

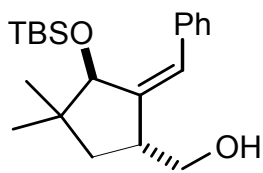

\author{
trans-2Z-benzylidene-1-tert-Butyldimethylsiloxy-3-hydroxymethyl-5,5- \\ dimethylcyclopentane (entry 3 ). \\ The title compound was prepared from the corresponding enyne ( $322 \mathrm{mg}, 0.98$ \\ $\mathrm{mmol}$ ) according to the procedure described for $\mathbf{3 b}$ (colorless oil, $239 \mathrm{mg}, 70 \%$ ).
}

$\mathrm{R}_{f} 0.30$ (15\% EtOAc/hexanes); IR (thin film) v 3436, 1255, 1067, 734, $650 \mathrm{~cm}^{-1}$; ${ }^{1} \mathrm{H}$ NMR (400 MHz, $\left.\mathrm{CDCl}_{3}\right) \delta 7.34-7.28(\mathrm{~m}, 2 \mathrm{H}), 7.26-7.18(\mathrm{~m}, 3 \mathrm{H}), 6.43(\mathrm{~d}, \mathrm{~J}=2.4 \mathrm{~Hz}, 1 \mathrm{H}), 4.49(\mathrm{~s}, 1 \mathrm{H}), 3.91(\mathrm{dd}, \mathrm{J}=5.1$, $10.6 \mathrm{~Hz}, 1 \mathrm{H}), 3.72(\mathrm{dd}, \mathrm{J}=10.6,7.2 \mathrm{~Hz}, 1 \mathrm{H}), 3.11(\mathrm{~m}, 1 \mathrm{H}), 2.07(\mathrm{dd}, \mathrm{J}=12.0,12.0 \mathrm{~Hz}, 1 \mathrm{H}), 1.90$ (br s, 1H), 1.43 (dd, J = 4.1, $12.7 \mathrm{~Hz}, 1 \mathrm{H}), 1.09$ (s, 3H), 0.99 (s, 3H), 0.79 (s, 9H), -0.33 (s, 3H), -0.35 (s, 3H) ${ }^{13} \mathrm{C}$ NMR $\left(100 \mathrm{MHz}, \mathrm{CDCl}_{3}\right) \delta 149.5,138.1,128.5,128.1,126.5,122.8,79.3,65.8,42.5,41.6,40.0$, 27.4, 25.7, 23.5, 18.1, -5.0, -5.5; HRMS $m / z 347.23976$ (calcd for $\mathrm{C}_{21} \mathrm{H}_{35} \mathrm{O}_{2} \mathrm{Si}[\mathrm{M}+\mathrm{H}]^{+}, 347.24063$ ).

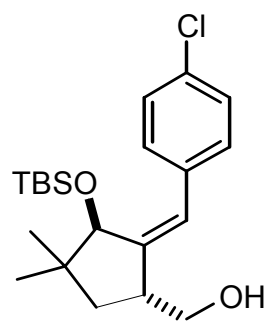

trans-1-tert-Butyldimethylsiloxy-2Z-(4-chlorophenyl)vinylidene-3hydroxymethyl-5,5-dimethylcyclopentane (entry 4).

The title compound was prepared from the corresponding enyne (360 mg, 0.99 $\mathrm{mmol}$ ) according to the procedure described for $\mathbf{3 b}$ (colorless oil, $226 \mathrm{mg}, 60 \%$ ).

$\mathrm{R}_{f} 0.57$ (25\% EtOAc/hexanes); IR (thin film) v 3329 (br), 1383, 1361, 1254, 1071 $\mathrm{cm}^{-1}$; ${ }^{1} \mathrm{H}$ NMR $\left(300 \mathrm{MHz}, \mathrm{CDCl}_{3}\right) \delta 7.26(\mathrm{~d}, \mathrm{~J}=8.5 \mathrm{~Hz}, 2 \mathrm{H}), 7.13(\mathrm{~d}, \mathrm{~J}=8.5 \mathrm{~Hz}$, $2 \mathrm{H}), 6.36(\mathrm{~d}, \mathrm{~J}=2.3 \mathrm{~Hz}, 1 \mathrm{H}), 4.38,(\mathrm{~s}, 1 \mathrm{H}), 3.87(\mathrm{dd}, \mathrm{J}=5.4,10.5 \mathrm{~Hz}, 1 \mathrm{H}), 3.70$ $(\mathrm{dd}, \mathrm{J}=6.9,10.5 \mathrm{~Hz}, 1 \mathrm{H}), 3.14-3.02(\mathrm{~m}, 1 \mathrm{H}), 2.04(\mathrm{dd}, \mathrm{J}=12.0,12.0 \mathrm{~Hz}, 1 \mathrm{H}), 1.53$ (br s, $1 \mathrm{H}), 1.37$ (dd, $\mathrm{J}=4.6,13.1 \mathrm{~Hz}, 1 \mathrm{H}), 1.05(\mathrm{~s}, 3 \mathrm{H}), 0.94(\mathrm{~s}, 3 \mathrm{H}), 0.76(\mathrm{~s}, 9 \mathrm{H}),-0.31(\mathrm{~s}, 3 \mathrm{H}),-0.38(\mathrm{~s}, 3 \mathrm{H}) ;{ }^{13} \mathrm{C}$ NMR $(75$ 
$\left.\mathrm{MHz}, \mathrm{CDCl}_{3}\right) \delta 150.4,136.6,132.2,129.8,128.3,121.5,79.3,65.8,42.6,41.6,39.9,27.4,25.7,23.4$, 18.1, -4.9, -5.2; HRMS m/z 380.19484 (calcd for $\mathrm{C}_{21} \mathrm{H}_{33} \mathrm{O}_{2} \mathrm{SiCl}[\mathrm{M}]^{+}, 380.19384$ ).

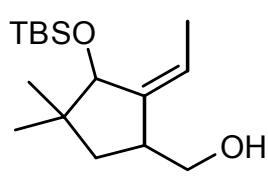

\section{1-tert-Butyldimethylsiloxy-2Z-ethylidine-3-hydroxymethyl-5,5- dimethylcyclopentane (entry 5).}

The title compound was prepared from the corresponding enyne $(262 \mathrm{mg}, 0.98$ $\mathrm{mmol}$ ) according to the procedure described for $\mathbf{3 b}$ as a mixture of cis/trans isomers (1:1.5 dr) (colorless oil, $213 \mathrm{mg}, 76 \%)$.

trans: $\mathrm{R}_{f} 0.63$ (25\% EtOAc/hexanes); IR (thin film) v 3332 (br), 1382, 1361, 1251, $1079 \mathrm{~cm}^{-1}$; ${ }^{1} \mathrm{H}$ NMR $\left(300 \mathrm{MHz}, \mathrm{CDCl}_{3}\right) \delta 5.29(\mathrm{q}, \mathrm{J}=6.9 \mathrm{~Hz}, 1 \mathrm{H}), 4.05(\mathrm{~s}, 1 \mathrm{H}), 3.72(\mathrm{dd}, \mathrm{J}=5.1,10.5 \mathrm{~Hz}, 1 \mathrm{H}), 3.55(\mathrm{dd}, \mathrm{J}=$ $6.7,10.5 \mathrm{~Hz}, 1 \mathrm{H}), 2.89-2.75(\mathrm{~m}, 1 \mathrm{H}), 1.87(\mathrm{dd}, \mathrm{J}=11.0,12.8 \mathrm{~Hz}, 1 \mathrm{H}), 1.70(\mathrm{dd}, \mathrm{J}=2.6,6.9 \mathrm{~Hz}, 3 \mathrm{H})$, 1.44 (br s, 1H), 1.24 (dd, J = 5.4, $12.8 \mathrm{~Hz}), 0.98$ (s, 3H), 0.85 (s, 9H), 0.77 (s, 3H), 0.05 (s, 3H), 0.00 (s, $3 \mathrm{H}) ;{ }^{13} \mathrm{C}$ NMR $\left(75 \mathrm{MHz}, \mathrm{CDCl}_{3}\right) \delta 145.8,117.0,79.9,65.7,42.2,41.2,39.8,27.2,25.8,23.3,18.2$, 14.9, -4.36, -4.44; HRMS $m / z$ 285.22458 (calcd for $\mathrm{C}_{16} \mathrm{H}_{33} \mathrm{O}_{2} \mathrm{Si}[\mathrm{M}+\mathrm{H}]^{+}, 285.22498$ ).
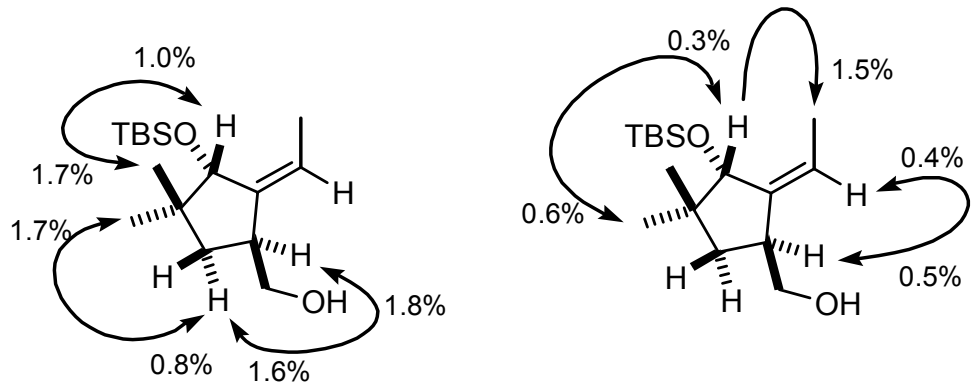

cis: $\mathrm{R}_{f} 0.73$ (25\% EtOAc/hexanes); IR (thin film) v 3354 (br),1382, 1360, 1251, $1072 \mathrm{~cm}^{-1}$; ${ }^{1} \mathrm{H}$ NMR $\left(300 \mathrm{MHz}, \mathrm{CDCl}_{3}\right) \delta 5.44(\mathrm{q}, \mathrm{J}=6.9 \mathrm{~Hz}, 1 \mathrm{H}), 3.96(\mathrm{~s}, 1 \mathrm{H}) 3.60(\mathrm{dd}, \mathrm{J}=4.4,9.7 \mathrm{~Hz}, 1 \mathrm{H}), 3.54-3.46(\mathrm{~m}$, $1 \mathrm{H}), 2.72-2.61(\mathrm{~m}, 1 \mathrm{H}), 2.39(\mathrm{br} \mathrm{s}, 1 \mathrm{H}), 1.73-1.63(\mathrm{~m}, 4 \mathrm{H}), 1.45(\mathrm{dd}, \mathrm{J}=8.97,12.81 \mathrm{~Hz}, 1 \mathrm{H}), 1.01(\mathrm{~s}$, $3 \mathrm{H}), 0.86(\mathrm{~s}, 9 \mathrm{H}), 0.76(\mathrm{~s}, 3 \mathrm{H}), 0.10(\mathrm{~s}, 3 \mathrm{H}), 0.04(\mathrm{~s}, 3 \mathrm{H}) ;{ }^{13} \mathrm{C} \mathrm{NMR}\left(75 \mathrm{MHz}, \mathrm{CDCl}_{3}\right) \delta 146.0,121.6$, 78.3, 66.7, 44.3, 42.9, 38.0, 26.0, 25.9, 23.3, 18.2, 15.6, -4.2, -4.5; HRMS $m / z$ 285.22500 (calcd for $\left.\mathrm{C}_{16} \mathrm{H}_{33} \mathrm{O}_{2} \mathrm{Si}[\mathrm{M}+\mathrm{H}]^{+}, 285.22498\right)$.
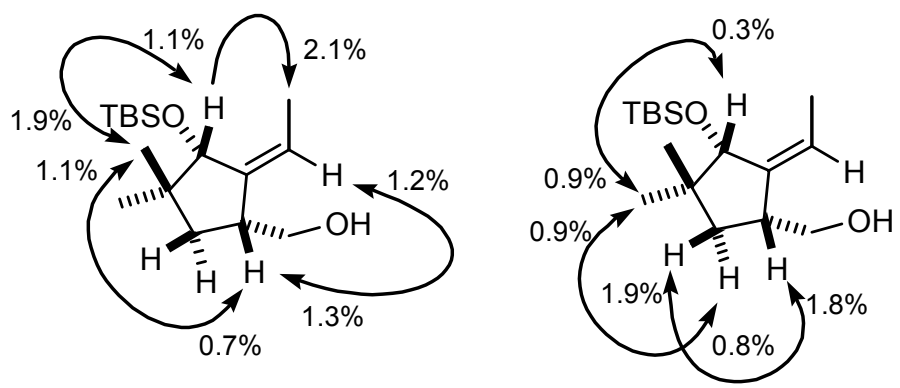

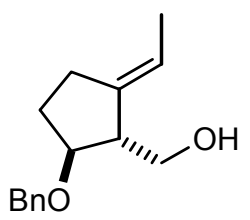

trans-1-Benzyloxy-3E-ethylidene-2-hydroxymethylcyclopentane (entry 6).

The title compound was prepared from the corresponding enyne $(211 \mathrm{mg}, 0.99$ $\mathrm{mmol}$ ) according to the procedure described for $\mathbf{3 b}$ as a mixture of cis/trans isomers (1:8 dr) (colorless oil, $147 \mathrm{mg}, 64 \%)$. 
trans: $\mathrm{R}_{f} 0.37$ (25\% EtOAc/hexanes); IR (thin film) v 3418 (br), 3031, 1095, 735, $697 \mathrm{~cm}^{-1}$; ${ }^{1} \mathrm{H}$ NMR $\left(300 \mathrm{MHz}, \mathrm{CDCl}_{3}\right) \delta$ 7.36-7.25 (m, 5H), 5.31 (dddq, J = 2.2, 2.3, 2.6, 6.7 Hz, 1H), $4.61(\mathrm{~d}, \mathrm{~J}=11.8 \mathrm{~Hz}$, $1 \mathrm{H}), 4.52(\mathrm{~d}, \mathrm{~J}=11.8 \mathrm{~Hz}, 1 \mathrm{H}), 3.87(\mathrm{ddd}, \mathrm{J}=6.2,6.2,6.2 \mathrm{~Hz}, 1 \mathrm{H}), 3.70(\mathrm{dd}, \mathrm{J}=4.9,10.8 \mathrm{~Hz}, 1 \mathrm{H})$, 2.70-2.59 (m, 1H), 2.51-2.36 (m, 1H), 2.25-2.10 (m, 1H), 2.09-1.92 (m, 1H), 1.78-1.65 (m, 1H), 1.65-1.57 (m, 3H); ${ }^{13} \mathrm{C} \mathrm{NMR}\left(75 \mathrm{MHz}, \mathrm{CDCl}_{3}\right) \delta 139.8,138.4,128.4,127.6,127.6,117.2,82.9,71.1$, 63.8, 51.9, 29.4, 26.1, 14.3; HRMS $m / z 233.15421$ (calcd for $\mathrm{C}_{15} \mathrm{H}_{21} \mathrm{O}_{2}[\mathrm{M}+\mathrm{H}]^{+}, 233.15416$ ).

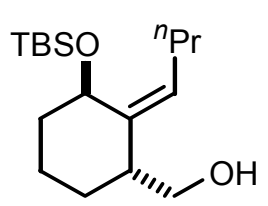

\section{trans-2Z-butylidene-1-tert-Butyldimethylsiloxy-3-hydroxymethylcyclohexane (entry 7).}

The title compound was prepared from the corresponding enyne ( $276 \mathrm{mg}, 0.99$ $\mathrm{mmol}$ ) according to the procedure described for $\mathbf{3 b}$ (colorless oil, $147 \mathrm{mg}, 49 \%$ ).

$\mathrm{R}_{f} 0.32$ (10\% EtOAc/hexanes); IR (thin film) v 3316 (br), 1252, $1037 \mathrm{~cm}^{-1} ;{ }^{1} \mathrm{H}$ NMR (400 MHz, $\left.\mathrm{CDCl}_{3}\right) \delta 4.92(\mathrm{dddd}, \mathrm{J}=1.0,7.2,7.2,7.2 \mathrm{~Hz}, 1 \mathrm{H}), 4.73(\mathrm{ddd}, 2.7,2.7,2.7 \mathrm{~Hz}, 1 \mathrm{H}), 3.77(\mathrm{dd}, \mathrm{J}=6.5$, $10.6 \mathrm{~Hz}, 1 \mathrm{H}), 3.54(\mathrm{dd}, \mathrm{J}=6.2,10.6 \mathrm{~Hz}, 1 \mathrm{H}), 2.66-2.56(\mathrm{~m}, 1 \mathrm{H}), 2.03(\operatorname{app~dq}, \mathrm{J}=1.0,7.4 \mathrm{~Hz}, 2 \mathrm{H})$, 1.96-1.75 (m, 3H), 1.53-1.45 (m, 1H), 1.43-1.22, (m, 4H), 1.01-0.92 (m, 1H) 0.88 (dd, J = 3.4, $3.4 \mathrm{~Hz}$, $3 \mathrm{H}), 0.86(\mathrm{~s}, 9 \mathrm{H}), 0.02(\mathrm{~s}, 3 \mathrm{H}),-0.03(\mathrm{~s}, 3 \mathrm{H}) ;{ }^{13} \mathrm{C} \mathrm{NMR}\left(100 \mathrm{MHz}, \mathrm{CDCl}_{3}\right) \delta 142.1,119.3,65.9,64.9$, 40.1, 35.9, 31.9, 29.1, 25.8, 23.3, 20.0, 18.0, 13.9, -4.6, -4.9; HRMS $m / z 299.24056$ (calcd for $\left.\mathrm{C}_{17} \mathrm{H}_{35} \mathrm{O}_{2} \mathrm{Si}[\mathrm{M}+\mathrm{H}]^{+}, 299.24063\right)$.

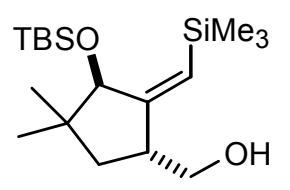

trans-1-tert-Butyldimethylsiloxy-3-hydroxymethyl-5,5-dimethyl-2Ztrimethylsilylvinylidenecyclopentane (8).

The title compound was prepared from the corresponding enyne ( $315 \mathrm{mg}, 0.97$ mmol) according to the procedure described for $\mathbf{3 b}$ except a solution of $\mathrm{NaOOH}$ prepared from $\mathrm{NaOH}(2 \mathrm{~mL}, 6 \mathrm{M}, 12 \mathrm{mmol})$ and $\mathrm{H}_{2} \mathrm{O}_{2}(4 \mathrm{~mL}, 30 \%, 35 \mathrm{mmol})$ was used for oxidation (white solid, $176 \mathrm{mg}, 53 \%$ ).

$\mathrm{R}_{f} 0.36$ (10\% EtOAc/hexanes); IR (thin film) v 3318 (br), 1635, 1249, $1078 \mathrm{~cm}^{-1}$; ${ }^{1} \mathrm{H} \mathrm{NMR}(400 \mathrm{MHz}$ $\left.\mathrm{CDCl}_{3}\right) \delta 5.26(\mathrm{~d}, \mathrm{~J}=2.4 \mathrm{~Hz}, 1 \mathrm{H}), 3.90(\mathrm{~s}, 1 \mathrm{H}), 3.79(\mathrm{dd}, \mathrm{J}=5.1,10.9 \mathrm{~Hz}, 1 \mathrm{H}), 3.61(\mathrm{dd}, \mathrm{J}=6.5,10.9$ $\mathrm{Hz}, 1 \mathrm{H}), 3.02-2.91(\mathrm{~m}, 1 \mathrm{H}), 1.92(\mathrm{dd}, \mathrm{J}=12.1,12.1 \mathrm{~Hz}, 1 \mathrm{H}), 1.41$ (br s, $1 \mathrm{H}), 1.24$ (dd, J = 4.8, $12.7 \mathrm{~Hz}$, $1 \mathrm{H}), 1.02(\mathrm{~s}, 3 \mathrm{H}), 0.85(\mathrm{~s}, 9 \mathrm{H}), 0.74(\mathrm{~s}, 3 \mathrm{H}), 0.12(\mathrm{~s}, 9 \mathrm{H}), 0.06(\mathrm{~s}, 3 \mathrm{H}), 0.02(\mathrm{~s}, 3 \mathrm{H}) ;{ }^{13} \mathrm{C} \mathrm{NMR}(100$ $\left.\mathrm{MHz} \mathrm{CDCl}_{3}\right) \delta 162.8,120.1,82.9,65.0,43.8,40.5,39.3,27.4,25.8,23.7,18.4,0.6,-3.8,-4.3$; HRMS $m / z 343.24592$ (calcd for $\mathrm{C}_{18} \mathrm{H}_{39} \mathrm{O}_{2} \mathrm{Si}_{2}[\mathrm{M}+\mathrm{H}]^{+}, 343.24886$ ).

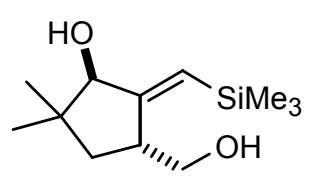

\section{trans-1-hydroxy-3-hydroxymethyl-5,5-dimethyl-2Z-} trimethylsilylvinylidenecyclopentane (9).

The title compound accompanied $\mathbf{8}$ in the cyclization/oxidation reaction (white solid, $69 \mathrm{mg}, 31 \%$ ).

$\mathrm{R}_{f} 0.44$ (30 \% EtOAc/hexanes); IR (thin film) v 3276 (br), 1629, 1245, $1138 \mathrm{~cm}^{-1}$; ${ }^{1} \mathrm{H}$ NMR (400 MHz, $\left.\mathrm{CDCl}_{3}\right) \delta 5.59(\mathrm{dd}, \mathrm{J}=2.2,2.2 \mathrm{~Hz}, 1 \mathrm{H}), 3.86(\mathrm{br} \mathrm{s}, 1 \mathrm{H}), 3.61(\mathrm{dd}, \mathrm{J}=4.8,18.5 \mathrm{~Hz}, 1 \mathrm{H}), 3.58(\mathrm{dd}, \mathrm{J}=$ $6.8,18.5 \mathrm{~Hz}, 1 \mathrm{H}), 2.84-2.74(\mathrm{~m}, 1 \mathrm{H}), 1.71(\mathrm{dd}, \mathrm{J}=8.9,13.0 \mathrm{~Hz}, 1 \mathrm{H}), 1.39$ (dd, J = 8.6, $13.0 \mathrm{~Hz}, 1 \mathrm{H})$, 1.65-1.44 (br s, 2H), 1.10 (s, 3H), $0.68(\mathrm{~s}, 3 \mathrm{H}), 0.13(\mathrm{~s}, 9 \mathrm{H}) ;{ }^{13} \mathrm{C} \mathrm{NMR}\left(100 \mathrm{MHz}, \mathrm{CDCl}_{3}\right) \delta 162.4$, 120.0, 82.9, 67.1, 40.5, 40.0, 38.9, 26.4, 19.6, 0.1; HRMS $m / z 227.14715$ (calcd for $\mathrm{C}_{12} \mathrm{H}_{23} \mathrm{O}_{2} \mathrm{Si}[\mathrm{M}-\mathrm{H}]^{+}$, 227.14673). 


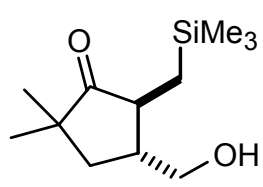

\section{trans-2-trimethylsilylmethyl-3-hydroxymethyl-5,5-dimethylcyclopentanone} (12).

A solution of diol $9(12 \mathrm{mg})$ in $\mathrm{CDCl}_{3}(0.75 \mathrm{~mL})$ was allowed to stand at $\mathrm{rt}$ for several days. Concentration and flash chromatography of the residue on $\mathrm{SiO}_{2}$ (elution gradient: $5 \%$ EtOAc/hexanes to $30 \%$ EtOAc/hexanes) provided the title compound as a white solid (11 mg, 92\%).

$\mathrm{R}_{f} 0.47$ (30\% EtOAc/hexanes); IR (thin film) v 3458 (br), 1737, $1248 \mathrm{~cm}^{-1}$; ${ }^{1} \mathrm{H}$ NMR (400 MHz, $\left.\mathrm{CDCl}_{3}\right) \delta 3.84(\mathrm{dd}, \mathrm{J}=3.3,10.8 \mathrm{~Hz}, 1 \mathrm{H}), 3.64(\mathrm{dd}, \mathrm{J}=5.6,10.8 \mathrm{~Hz}, 1 \mathrm{H}), 2.11-1.8(\mathrm{~m}, 3 \mathrm{H}), 1.52$ (ddd, J $=2.6,12.8,12.8 \mathrm{~Hz}, 1 \mathrm{H}), 1.60-1.45(\mathrm{br} \mathrm{s}, 1 \mathrm{H}), 1.08(\mathrm{~s}, 3 \mathrm{H}), 1.00(\mathrm{~s}, 3 \mathrm{H}), 0.81(\mathrm{dd}, \mathrm{J}=7.9,14.9 \mathrm{~Hz}, 1 \mathrm{H})$, $0.69(\mathrm{dd}, \mathrm{J}=4.4,14.9 \mathrm{~Hz}, 1 \mathrm{H}) 0.02(\mathrm{~s}, 9 \mathrm{H}) ;{ }^{13} \mathrm{C} \mathrm{NMR}\left(100 \mathrm{MHz}, \mathrm{CDCl}_{3}\right) \delta 224.0,64.7,47.2,44.7$, 43.9, 40.1, 25.4, 25.0, 16.5, -0.7; HRMS m/z 228.15476 (calcd for $\mathrm{C}_{12} \mathrm{H}_{24} \mathrm{O}_{2} \mathrm{Si}[\mathrm{M}]^{+}, 228.15456$ ).

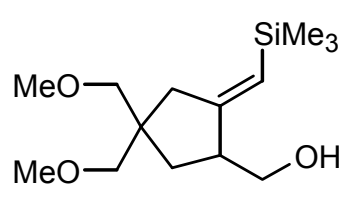

\section{E-trimethylsilylvinylidine-4-hydroxymethyl-1,1-} bis(methoxymethyl)cyclopentane (entry 9).

The title compound was prepared from the corresponding enyne $(250 \mathrm{mg}, 0.98$ $\mathrm{mmol}$ ) according to the procedure described for $\mathbf{3 b}$ except a solution of $\mathrm{NaOOH}$ prepared from $\mathrm{NaOH}(2 \mathrm{~mL}, 6 \mathrm{M}, 12 \mathrm{mmol})$ and $\mathrm{H}_{2} \mathrm{O}_{2}(4 \mathrm{~mL}, 30 \%$, $35 \mathrm{mmol}$ ) was used for oxidation (colorless liquid, $171 \mathrm{mg}, 64 \%$ ).

$\mathrm{R}_{f} 0.30$ (25\% EtOAc/hexanes); IR (thin film) v 3447 (br), 1653, $1107 \mathrm{~cm}^{-1} ;{ }^{1} \mathrm{H}$ NMR (300 MHz, $\left.\mathrm{CDCl}_{3}\right) \delta 5.40(\mathrm{ddd}, \mathrm{J}=1.8,2.1,2.1 \mathrm{~Hz}, 1 \mathrm{H}), 3.68-3.58(\mathrm{~m}, 2 \mathrm{H}), 3.35(\mathrm{~s}, 3 \mathrm{H}), 3.33(\mathrm{~s}, 3 \mathrm{H}), 3.30(\mathrm{~s}, 2 \mathrm{H})$, $3.20(\mathrm{~d}, \mathrm{~J}=12.6 \mathrm{~Hz}, 1 \mathrm{H}), 3.17(\mathrm{~d}, \mathrm{~J}=12.6 \mathrm{~Hz}, 1 \mathrm{H}), 2.74-2.62(\mathrm{~m}, 1 \mathrm{H}), 2.35(\mathrm{~d}, \mathrm{~J}=16.4 \mathrm{~Hz}, 1 \mathrm{H}), 2.21$ (dd, J =2.1, $16.4 \mathrm{~Hz}, 1 \mathrm{H}), 1.82$ (dd, J = 8.7, $13.3 \mathrm{~Hz}, 1 \mathrm{H}), 1.74$ (br s, $1 \mathrm{H}), 1.43$ (dd, J = 8.7, $13.3 \mathrm{~Hz}$, $1 \mathrm{H}), 0.10(\mathrm{~s}, 9 \mathrm{H}) ;{ }^{13} \mathrm{C} \mathrm{NMR}\left(75 \mathrm{MHz}, \mathrm{CDCl}_{3}\right) \delta 161.2,121.0,77.1,75.2,65.4,59.2,59.2,47.6,46.4$, 39.2, 33.6, -0.3; HRMS $m / z 271.17251$ (calcd for $\mathrm{C}_{14} \mathrm{H}_{27} \mathrm{O}_{3} \mathrm{Si}[\mathrm{M}-\mathrm{H}]^{+}, 271.17295$ ).
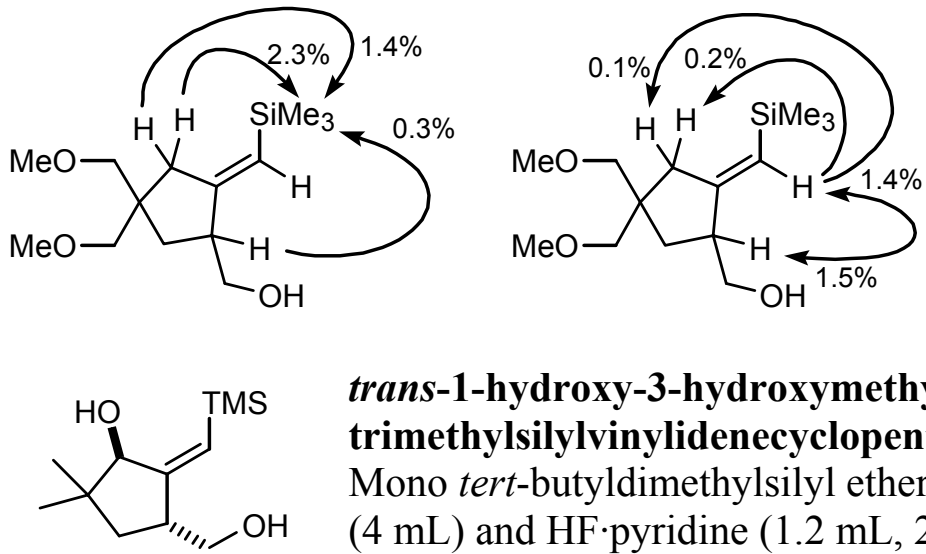

\section{trans-1-hydroxy-3-hydroxymethyl-5,5-dimethyl-2E-} trimethylsilylvinylidenecyclopentane (10).

Mono tert-butyldimethylsilyl ether $8(0.072,0.21 \mathrm{mmol})$ was dissolved in $\mathrm{MeOH}$ (4 mL) and HF.pyridine $(1.2 \mathrm{~mL}, 20 \%$, xx mmol) was added. After heating the mixture at $60{ }^{\circ} \mathrm{C}$ for $24 \mathrm{~h}, \mathrm{NaHCO}_{3}$ (sat'd, $15 \mathrm{~mL}$ ) was added. The mixture was extracted with EtOAc $(3 \times 15 \mathrm{~mL})$ and the combined extracts were washed with brine, dried (MgSO4), filtered and concentrated. Purification of the residue by flash chromatography on $\mathrm{SiO}_{2}$ (elution gradient: $5 \% \mathrm{EtOAc} /$ hexanes to $30 \% \mathrm{EtOAc} /$ hexanes) provided the title compound as a white solid (16 $\mathrm{mg}$, $33 \%$ ). The starting silyl ether 8 was also recovered (14 mg, 19\%)

$\mathrm{R}_{f} 0.44$ (30\% EtOAc/hexanes); IR (thin film) v 3385 (br), 1626, 1246, $1088 \mathrm{~cm}^{-1}$; ${ }^{1} \mathrm{H}$ NMR (400 MHz, $\left.\mathrm{CDCl}_{3}\right) \delta 5.55(\mathrm{dd}, \mathrm{J}=2.4,2.4 \mathrm{~Hz}, 1 \mathrm{H}), 4.00(\operatorname{app~d}, \mathrm{J}=7.9 \mathrm{~Hz}, 1 \mathrm{H}), 3.62(\operatorname{app~d}, \mathrm{J}=5.5 \mathrm{~Hz}, 1 \mathrm{H}), 2.75-$ 
$2.65(\mathrm{~m}, 1 \mathrm{H}), 1.88(\mathrm{dd}, \mathrm{J}=8.9,13.0 \mathrm{~Hz}, 1 \mathrm{H}), 1.38(\mathrm{br} \mathrm{s}, 1 \mathrm{H}), 1.35(\mathrm{dd}, \mathrm{J}=9.6,13.0 \mathrm{~Hz}, 1 \mathrm{H}), 1.03(\mathrm{~s}$, $3 \mathrm{H}), 0.85(\mathrm{~s}, 3 \mathrm{H}), 0.12(\mathrm{~s}, 9 \mathrm{H}) ;{ }^{13} \mathrm{C} \mathrm{NMR}\left(100 \mathrm{MHz}, \mathrm{CDCl}_{3}\right) \delta 163.3,124.6,83.1,66.0,44.2,40.5,39.2$, 26.7, 20.4, 0.7; HRMS $m / z$ 229, 16238 (calcd for $\mathrm{C}_{12} \mathrm{H}_{25} \mathrm{O}_{2} \mathrm{Si}[\mathrm{M}+\mathrm{H}]^{+}$, 227.14673).

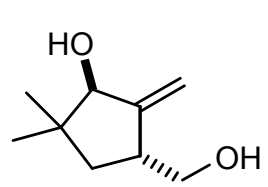

trans-1-hydroxy-3-hydroxymethyl-5,5-dimethylvinylidenecyclopentane. The title compound accompanied $\mathbf{1 0}$ in the deprotection of tert-butyldimethylsilyl ether 8 (15 mg, 46\%).

$\mathrm{R}_{f} 0.16$ (30\% EtOAc/hexanes); IR (thin film) v 3349, 1662, 1092, $1029 \mathrm{~cm}^{-1} ;{ }^{1} \mathrm{H}$ $\operatorname{NMR}\left(400 \mathrm{MHz}, \mathrm{CDCl}_{3}\right) \delta 5.18(\mathrm{dd}, \mathrm{J}=2.7,2.7 \mathrm{~Hz}, 1 \mathrm{H}), 5.07(\mathrm{dd}, \mathrm{J}=2.7,2.7 \mathrm{~Hz}, 1 \mathrm{H}), 3.94$ (br s, $1 \mathrm{H}), 3.62(\mathrm{dd}, \mathrm{J}=6.2,10.6 \mathrm{~Hz}, 1 \mathrm{H}), 3.53(\mathrm{dd}, \mathrm{J}=5.8 \mathrm{~Hz}, 10.9 \mathrm{~Hz}), 2.75-2.64(\mathrm{~m}, 1 \mathrm{H}), 1.67(\mathrm{dd}, \mathrm{J}=8.5$, $13.0 \mathrm{~Hz}, 1 \mathrm{H}$ ), 1.52 (br s, 1H), 1.40 (br s, 1H), 1.27 (dd, J =9.6, $13.0 \mathrm{~Hz}, 1 \mathrm{H}), 1.08$ (s, $3 \mathrm{H}), 0.76$ (s, $3 \mathrm{H})$; ${ }^{13} \mathrm{C} \mathrm{NMR}\left(100 \mathrm{MHz}, \mathrm{CDCl}_{3}\right) \delta 154.7,107.8,82.2,66.2,41.2,40.5,38.6,26.2,19.5 ; \mathrm{HRMS} \mathrm{m} / z$ 155.10721 (calcd for $\mathrm{C}_{9} \mathrm{H}_{15} \mathrm{O}_{2}[\mathrm{M}-\mathrm{H}]^{+}, 227.14673$ ). 